\title{
Pulmonary Function should be the Surveillance Tool in Obese Children to Prevent Asthma
}

\section{Yungling L. Lee}

Institute of Epidemiology and Preventive Medicine, National Taiwan University, Taipei, Taiwan

Objective

To investigate the important mediator linking from obesity to childhood asthma

Introduction

The mediating pathways in the obesity-asthma link are largely unknown. We aimed to investigate the mediating pathways and to search for the most prominent pathological mechanism between central obesity and childhood asthma.

\section{Methods}

In Taiwan Children Health Study, we collected data on an open cohort of children ranged from 9 to 13 years old. Children's respiratory outcomes, atopic conditions, obesity measures, and pulmonary function were surveyed annually between 2010 and 2012. Fractional exhaled nitric oxide concentrations were recorded in 2012. Generalized estimating equations and general linear models were used to examine the associations among central obesity, possible mediators, and asthma. Structural equation models were applied to investigate the pathways that mediate the link between central obesity and asthma.

\section{Results}

Central obesity (waist-to-hip ratio) most accurately predicted childhood asthma. In the model of active asthma, the percentage of mediation was $28.6 \%$ for pulmonary function, $18.1 \%$ for atopy, and $5.7 \%$ for airway inflammation. The percentage of mediation for pulmonary function was $40.2 \%$ in the model of lifetime wheeze. Pulmonary function was responsible for the greatest percentage of mediation among the three mediators in both models.

\section{Conclusions}

Decline in pulmonary function is the most important pathway in central obesity-related asthma. Pulmonary function surveillance should be applied to obese children for asthma risk prediction. 